\title{
An Efficient Protocol for the Synthesis of O-Fluoroalkylisoureas through Copper-Catalysed, Three-Component Reaction of Cyan- amides, Fluoroalcohols and Diaryliodonium Triflates
}

\author{
Jihui Lia,b \\ Weiguang $Y u^{a, b}$ \\ Yifeng Hou ${ }^{\mathrm{a}, \mathrm{b}}$ \\ Wenxing Fu ${ }^{a, b}$ \\ Shuying $\mathrm{Xu}{ }^{* a, b}$ \\ Yucang Zhang*a,b \\ ${ }^{a}$ Key Laboratory of Ministry of Education for Advanced Materi- \\ als in Tropical Island Resources in Hainan University, Haikou \\ 570228, P. R. of China \\ ${ }^{\mathrm{b}}$ College of Materials and Chemical Engineering, Hainan Univer- \\ sity, Haikou 570228, P. R. of China \\ xushuying1980@hainu.edu.cn \\ yczhang@hainu.edu.cn
}

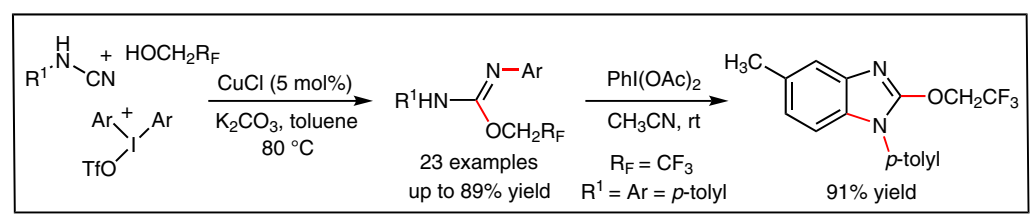

Received: 15.05.2017

Accepted after revision: 20.06.2017

Published online: 08.08 .2017

DOI: 10.1055/s-0036-1590828; Art ID: so-2017-d0019-op

License terms: CC

Abstract A copper-catalysed, three-component reaction involving cyanamides, fluoroalcohols and diaryliodonium triflates is disclosed for the synthesis of $\mathrm{O}$-fluoroalkylisoureas. Various $\mathrm{O}$-fluoroalkylisoureas were obtained in good yields by using simple and readily available substrates. Moreover, $\mathrm{C}-\mathrm{H}$ activation of $\mathrm{O}$-fluoroalkylisoureas mediated by $\mathrm{Phl}(\mathrm{OAC})_{2}$ was established to obtain 2-fluoroalkoxybenzimidazoles in high yields at room temperature.

Key words copper-catalysis, three-component reaction, O-fluoroalkylisourea, 2-alkoxybenzimidazole, $\mathrm{C}-\mathrm{H}$ activation

Isoureas, an important class of versatile organic reagent, have been widely used as guanylating and alkylating agents. ${ }^{1}$ Additionally, they are key precursors for constructing various bioactive molecules, such as the hypertension drug Candesartan, ${ }^{2}$ glucocerebrosidase inhibitor fused oxazolidin-2-imines and nanomolar enzyme activity enhancer spiro oxazolidin-2-imines. ${ }^{3}$ Despite their widespread applications, only a limited number of synthetic routes to isoureas have been reported, mainly relying on nucleophilic addition of alcohols to carbodiimides (Scheme 1, a). Initially, thermal acid- or base-promoted reactions were developed by Stieglitz ${ }^{4}$ and Dains, ${ }^{5}$ which are only suitable for the synthesis of $N$-arylisoureas. Subsequently, copper and zinc catalysts were used to promote the reaction by Däbritz ${ }^{6}$ and Schmidt, ${ }^{7}$ respectively, providing more efficient routes for the preparation of both $\mathrm{N}$-arylisoureas and $\mathrm{N}$-alkylisoureas. Recently, uranium and thorium amide catalysts have also been applied to the reaction, which is reported by Eisen to be a highly efficient synthetic protocol. ${ }^{8}$ These approaches are all similar, and more diverse methodologies involving simple and easily available starting materials are clearly required for the synthesis of isoureas.

Inspired by our work on transition-metal-catalysed multicomponent reactions for the synthesis of $\mathrm{N}$-molecules, ${ }^{9}$ we have recently developed a copper-catalysed, three-component reaction of cyanamides, amines and diaryliodoniums for the synthesis of guanidines. Here, an extension of this methodology to fluroloalcohols was explored for the rapid synthesis of 0 -fluoroalkylisoureas that can be useful synthons for potentially bioactive cyclic fluoro-isoureas ${ }^{10}$ (Scheme 1, b).

We started our study by investigating the reaction of $p$ tolylcyanamide (1a), di(p-tolyl)iodonium triflate (2a) and 2,2,2-trifluoroethanol (3a) in the presence of $\mathrm{K}_{2} \mathrm{CO}_{3}$ using $\mathrm{CuCl}$ ( $5 \mathrm{~mol} \%)$ as catalyst with bipy (2,2'-bipyridyl) in toluene at $80^{\circ} \mathrm{C}$ under $\mathrm{N}_{2}$ for $2 \mathrm{~h}$ (Table 1 , entry 1 ). Gratifyingly, the reaction afforded the desired isourea $4 a$ in $62 \%$ yield, and the reaction under air produced $4 \mathbf{a}$ in $47 \%$ yield (entry 2 ). The reaction conditions including bases, solvents, ligands, and copper catalysts were then screened in detail (Table 1). Other bases such as $\mathrm{NaHCO}_{3}, \mathrm{Cs}_{2} \mathrm{CO}_{3}, \mathrm{~K}_{3} \mathrm{PO}_{4}$,

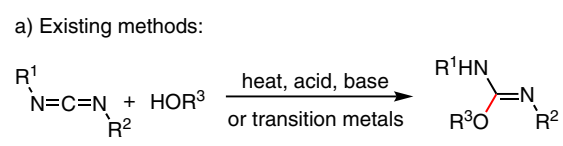

b) Present work:

Scheme 1 Approaches to the synthesis of isoureas 
t-BuOK and $\mathrm{Et}_{3} \mathrm{~N}$ all afforded inferior yields (entries 3-7), and the reaction without base did not produce any product (entry 8).

Table 1 Optimization of Reaction Conditions ${ }^{\mathrm{a}}$

\begin{tabular}{|c|c|c|c|c|c|}
\hline Entry & Base & Solvent & L & Cat. & Yield (\%) \\
\hline 1 & $\mathrm{~K}_{2} \mathrm{CO}_{3}$ & toluene & Bipy & $\mathrm{CuCl}$ & 62 \\
\hline $2^{c}$ & $\mathrm{~K}_{2} \mathrm{CO}_{3}$ & toluene & Bipy & $\mathrm{CuCl}$ & 47 \\
\hline 3 & $\mathrm{NaHCO}_{3}$ & toluene & Bipy & $\mathrm{CuCl}$ & 22 \\
\hline 4 & $\mathrm{Cs}_{2} \mathrm{CO}_{3}$ & toluene & Bipy & $\mathrm{CuCl}$ & 52 \\
\hline 5 & $\mathrm{~K}_{3} \mathrm{PO}_{4}$ & toluene & Bipy & $\mathrm{CuCl}$ & 33 \\
\hline 6 & $t$-BuOK & toluene & Bipy & $\mathrm{CuCl}$ & 46 \\
\hline 7 & $\mathrm{Et}_{3} \mathrm{~N}$ & toluene & Bipy & $\mathrm{CuCl}$ & 28 \\
\hline 8 & - & toluene & Bipy & $\mathrm{CuCl}$ & 0 \\
\hline 9 & $\mathrm{~K}_{2} \mathrm{CO}_{3}$ & dioxane & Bipy & $\mathrm{CuCl}$ & 26 \\
\hline 10 & $\mathrm{~K}_{2} \mathrm{CO}_{3}$ & DMSO & Bipy & $\mathrm{CuCl}$ & 20 \\
\hline 11 & $\mathrm{~K}_{2} \mathrm{CO}_{3}$ & $\mathrm{H}_{2} \mathrm{O}$ & Bipy & $\mathrm{CuCl}$ & 25 \\
\hline 12 & $\mathrm{~K}_{2} \mathrm{CO}_{3}$ & DMF & Bipy & $\mathrm{CuCl}$ & 22 \\
\hline 13 & $\mathrm{~K}_{2} \mathrm{CO}_{3}$ & THF & Bipy & $\mathrm{CuCl}$ & 31 \\
\hline 14 & $\mathrm{~K}_{2} \mathrm{CO}_{3}$ & toluene & 1,10-Phen & $\mathrm{CuCl}$ & 31 \\
\hline 15 & $\mathrm{~K}_{2} \mathrm{CO}_{3}$ & toluene & $\mathrm{PPh}_{3}$ & $\mathrm{CuCl}$ & 61 \\
\hline 16 & $\mathrm{~K}_{2} \mathrm{CO}_{3}$ & toluene & - & $\mathrm{CuCl}$ & 65 \\
\hline 17 & $\mathrm{~K}_{2} \mathrm{CO}_{3}$ & toluene & - & $\mathrm{CuBr}$ & 54 \\
\hline 18 & $\mathrm{~K}_{2} \mathrm{CO}_{3}$ & toluene & - & Cul & 53 \\
\hline $19^{d}$ & $\mathrm{~K}_{2} \mathrm{CO}_{3}$ & toluene & - & $\mathrm{CuCl}$ & 64 \\
\hline 20 & $\mathrm{~K}_{2} \mathrm{CO}_{3}$ & toluene & - & - & 0 \\
\hline $21^{e}$ & $\mathrm{~K}_{2} \mathrm{CO}_{3}$ & toluene & - & $\mathrm{CuCl}$ & 51 \\
\hline $22^{f}$ & $\mathrm{~K}_{2} \mathrm{CO}_{3}$ & toluene & - & $\mathrm{CuCl}$ & 48 \\
\hline
\end{tabular}

a Reaction conditions: $p$-tolylcyanamide $1 \mathbf{a}(0.3 \mathrm{mmol})$, di(p-tolyl)iodonium triflate $2 \mathrm{a}(0.3 \mathrm{mmol}), 2,2,2$-trifluoroethanol $3 \mathrm{a}(0.2 \mathrm{mmol})$, copper salt

$(0.01 \mathrm{mmol})$, solvent $(1.0 \mathrm{~mL})$, stirred under $\mathrm{N}_{2}, 80^{\circ} \mathrm{C}, 2 \mathrm{~h}$.

${ }^{\mathrm{b}}$ Isolated yield.

c The reaction was performed under air.

${ }^{d} \mathrm{CuCl}(0.02 \mathrm{mmol})$ was used.

e Ratio $1 \mathbf{a} / 2 \mathbf{2} / 3 \mathbf{a}=1: 1.5: 1.5$.

f Ratio $1 \mathrm{a} / 2 \mathrm{a} / 3 \mathbf{a}=1: 1: 1.5$

Replacing toluene with other solvents such as dioxane, DMSO, $\mathrm{H}_{2} \mathrm{O}$, DMF or THF did not improve the reaction yield (Table 1, entries 9-13). The reactions with 1,10-phen or $\mathrm{PPh}_{3}$ as ligands did not provide higher yields (entries 14 and $15)$, and the reaction without ligand afforded a slightly higher yield (entry 16). Other catalysts such as $\mathrm{CuBr}$ and $\mathrm{CuI}$ were inferior to $\mathrm{CuCl}$ (entries 17 and 18). Increasing the amount of $\mathrm{CuCl}$ (10 $\mathrm{mol} \%$ ) did not enhance the reaction yield (entry 19), and the absence of copper catalyst led to no desired isourea formation (entry 20). Finally, the sub- strate ratios $(\mathbf{1} \mathbf{a} / \mathbf{2} \mathbf{a} / \mathbf{3} \mathbf{a}=1.5: 1.5: 1,1: 1.5: 1.5,1: 1: 1.5)$ were explored (entries 16, 21 and 22), and the $\mathbf{1 a} / \mathbf{2 a} / \mathbf{3 a}=1.5: 1.5: 1$ ratio was found to be optimal.

With the optimised reaction conditions in hand, the scope of the reaction with respect to cyanamide was evaluated (Scheme 2). Phenylcyanamide gave $65 \%$ yield of $\mathbf{4 b}$, and $m$ - and $o$-tolyl cyanamides also formed the corresponding isoureas $\mathbf{4 c}$ and $\mathbf{4 d}$, respectively, in good yields. Arylcyanamides with either electron-withdrawing or electrondonating groups likewise provided $\mathbf{4 e}$ and $\mathbf{4 f}$ in comparably good yields. Impressively, 1-nathylcyanamide afforded the desired product $\mathbf{4 g}$ in high yield. Additionally, some aliphatic cyanamides were found to be suitable for the reaction. However, low yields were obtained for aliphatic cyanamides, as the cross-coupling products of the aliphatic cyanamides and $\mathrm{di}(p$-tolyl)iodonium triflate were found to be major products. ${ }^{11}$ For instance, benzylcyanamide and cyclohexylcyanamide both produced the corresponding isoureas 4h and $4 \mathbf{i}$ with $24 \%$ and $20 \%$ yield, respectively, and $t$-butylcyanamide did not afford any of desired product $\mathbf{4 j}$, which is probably due to the extreme steric hindrance of the $t$-butyl group.

A variety of the diaryliodonium triflates was then examined, as shown in Table 2. Symmetric diaryliodoniums with either electron-poor or electron-rich aryls produced the desired isoureas in good yields (entries 1-4). For example, $\operatorname{di}(p$-chrolophenyl)iodonium triflate and $\operatorname{di}[p$ - $(t-\mathrm{bu}-$ tyl)phenyl]iodonium triflate gave $63 \%$ and $55 \%$ yield, respectively (entries 2 and 4). The reactions of sterically hindered di(2,5-dimethylphenyl)iodonium triflate and di(2,4,6-trimethylphenyl)iodonium triflate furnished the desired products in higher yields (entries 5 and 6), because the side reactions between cyanamides and sterically hindered diaryliodoniums were reduced. For the unsymmetrical diaryliodonium triflates, the reactions provide good yields comparable to those with symmetrical diaryliodoniums triflates. The phenyliodonium triflates with $p$ - $(t-\mathrm{bu}-$ tyl)phenyl and $p$-iodophenyl both afforded a mixture of products with low chemoselectivities (entries 7 and 8). Interestingly, phenyl $\left(p-\mathrm{NO}_{2}\right.$-phenyl)iodonium only produced a single product $\mathbf{4 q}$ ' in low yield (entry 9). Phenyl(2,5-dimethylphenyl)iodonium triflate yielded two products $4 \mathbf{n}$ and $\mathbf{4 n}$ ' in 1.7:1 ratio (entry 10 ). These results suggest that the more electron-rich and bulkier aryl groups of the unsymmetrical diaryliodoniums were preferable transferred in this three-component reaction.

Finally, the scope of the reaction with respect to alcohol was explored (Scheme 3). We were pleased to find that the protocol was tolerant of many fluorine-substituted alcohols. Both mono- and di-fluoroethanols delivered the corresponding products $\mathbf{4 r}$ and $\mathbf{4 s}$ in $49 \%$ and $63 \%$ yield, respectively. 1,1,1,3,3,3-Hexafluoro-2-propanol furnished the desired product $4 \mathbf{t}$ in moderate yield (51\%). 

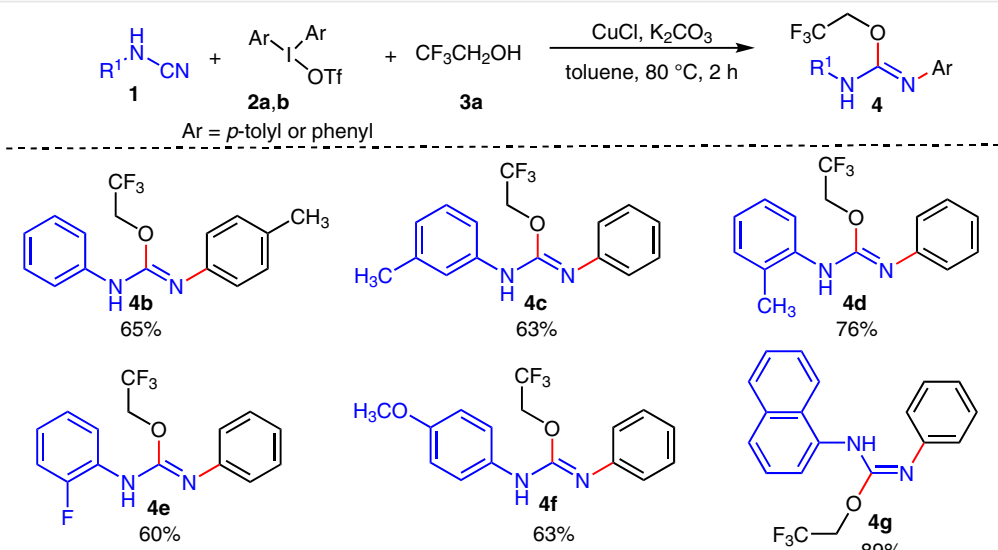<smiles>COc1ccc(NC(=Nc2ccccc2)OC(F)(F)F)cc1</smiles><smiles>FC(F)(F)COC(=Nc1ccccc1)Nc1cccc2ccccc12</smiles><smiles>FC(F)(F)COC(=Nc1ccccc1)NCc1ccccc1</smiles><smiles>FC(F)(F)COC(=Nc1ccccc1)NC1CCCCC1</smiles><smiles>Cc1ccc(/N=C(\NC(C)(C)C)OCC(F)(F)F)cc1</smiles>

Scheme $\mathbf{2}$ Scope of the reaction with respect to cyanamide. Reagents and conditions: Cyanamide $\mathbf{1} /$ diaryliodonium triflate (2a or $\mathbf{2 b}$ )/2,2,2-trifluoroethanol 3a [ $\mathbf{1} / \mathbf{2 a}$ (or $\mathbf{2 b}) / \mathbf{3} \mathbf{a}=1.5: 1.5: 1.0]$, $\mathrm{CuCl}$ (0.05 equiv), $\mathrm{K}_{2} \mathrm{CO}_{3}$ (2.25 equiv).

Table 2 Scope of the Reaction with Respect to Diaryliodonium Triflate ${ }^{a}$

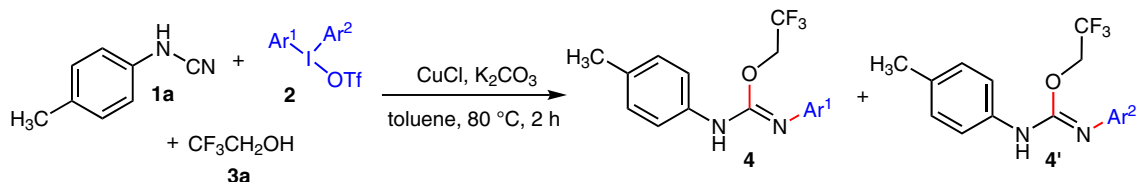

\begin{tabular}{|c|c|c|c|c|}
\hline Entry & $\mathrm{Ar}^{1}$ & $\mathrm{Ar}^{2}$ & $\mathbf{4} / \mathbf{4}^{\prime}$ (ratio) & Yield (\%) \\
\hline 1 & $\mathrm{C}_{6} \mathrm{H}_{5}$ & $\mathrm{C}_{6} \mathrm{H}_{5}$ & $4 b /-(-)$ & 67 \\
\hline 2 & $4-\mathrm{ClC}_{6} \mathrm{H}_{4}$ & $4-\mathrm{ClC}_{6} \mathrm{H}_{4}$ & $4 \mathbf{k} /-(-)$ & 63 \\
\hline 3 & 4- $\mathrm{BrC}_{6} \mathrm{H}_{4}$ & 4- $\mathrm{BrC}_{6} \mathrm{H}_{4}$ & $4 \mathrm{I} /-(-)$ & 35 \\
\hline 4 & 4-(t-butyl) $\mathrm{C}_{6} \mathrm{H}_{4}$ & 4-(t-butyl) $\mathrm{C}_{6} \mathrm{H}_{4}$ & $4 m /-(-)$ & 55 \\
\hline 5 & $2,5-\left(\mathrm{CH}_{3}\right)_{2} \mathrm{C}_{6} \mathrm{H}_{3}$ & $2,5-\left(\mathrm{CH}_{3}\right)_{2} \mathrm{C}_{6} \mathrm{H}_{3}$ & $4 n /-(-)$ & 76 \\
\hline 6 & $2,4,6-\left(\mathrm{CH}_{3}\right)_{3} \mathrm{C}_{6} \mathrm{H}_{2}$ & $2,4,6-\left(\mathrm{CH}_{3}\right)_{3} \mathrm{C}_{6} \mathrm{H}_{2}$ & $40 /-(-)$ & 73 \\
\hline 7 & 4-(t-butyl) $\mathrm{C}_{6} \mathrm{H}_{4}$ & $\mathrm{C}_{6} \mathrm{H}_{5}$ & $4 m / 4 m^{\prime}(1.5: 1)^{c}$ & 64 \\
\hline 8 & $4-\mathrm{IC}_{6} \mathrm{H}_{4}$ & $\mathrm{C}_{6} \mathrm{H}_{5}$ & $\mathbf{4} \mathbf{p} / \mathbf{4} \mathbf{p}^{\prime}(1: 1.1)^{c}$ & 61 \\
\hline 9 & $4-\mathrm{NO}_{2} \mathrm{C}_{6} \mathrm{H}_{4}$ & $\mathrm{C}_{6} \mathrm{H}_{5}$ & $\mathbf{4 q} / \mathbf{4} \mathbf{q}^{\prime}(0: 1)$ & 32 \\
\hline 10 & 2,5- $\left(\mathrm{CH}_{3}\right)_{2} \mathrm{C}_{6} \mathrm{H}_{3}$ & $\mathrm{C}_{6} \mathrm{H}_{5}$ & $\mathbf{4 n} / \mathbf{4} n^{\prime}(1.7: 1)$ & 70 \\
\hline
\end{tabular}

${ }^{a}$ Reaction conditions: -tolylcyanamide $\mathbf{1 a}$ /diaryliodonium triflate 2/2,2,2-trifluoroethanol (3a) (1a/2/3a=1.5:1.5:1.0), $\mathrm{CuCl}$ (0.05 equiv), $\mathrm{K}_{2} \mathrm{CO}_{3}(2.25$ equiv). b Isolated yield.

c Ratio based on ${ }^{1} \mathrm{H}$ NMR spectroscopic analysis.

Moreover, ethanol was found to be suitable for the reaction, although a low yield of $\mathbf{4} \mathbf{u}$ was obtained (34\%), indicating that fluorine plays an important role in activation of the alcohol ( $4 \mathbf{r}$ vs. $4 \mathbf{s}$ vs. $4 \mathbf{u}$ ). The reaction of phenol was complex, with formation of unidentified products.
Considering 2-alkoxybenzimidazole is the core of the hypertension drug candesartan, ${ }^{2}$ the $\mathrm{C}-\mathrm{H}$ activation of the isoureas was explored for the synthesis of 2-fluoroalkoxybenzimidazoles, which may have interesting bioactivities (Scheme 4). ${ }^{10}$ 
<smiles>Cc1ccc(N=C(Nc2ccc(C)cc2)OCP)cc1</smiles><smiles>CCOC(=Nc1ccc(C)cc1)Nc1ccc(C)cc1</smiles><smiles>CCOC(=Nc1ccc(C)cc1)Nc1ccc(C)cc1</smiles>

Scheme 3 Scope of the reaction with respect to fluoroalcohol. Reagents and conditions: $p$-tolylcyanamide $\mathbf{1 a} / \mathrm{di}(p$-tolyl)iodonium triflate 2a/alcohol 3 (1a/2a/3=1.5:1.5:1.0), $\mathrm{CuCl}$ (0.05 equiv), $\mathrm{K}_{2} \mathrm{CO}_{3}(2.25$ equiv). ${ }^{a}$ With di(p-tolyl)cyanamide, yield based on ${ }^{1} \mathrm{H}$ NMR spectroscopic analysis.

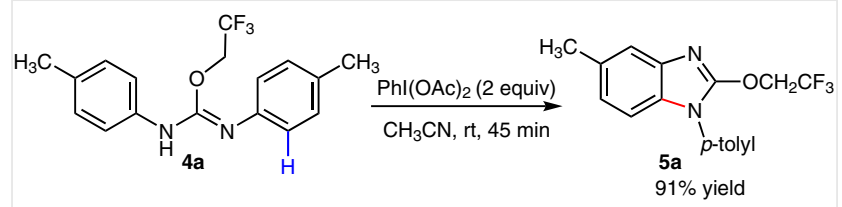

Scheme $4 \mathrm{Phl}(\mathrm{OAc})_{2}$-promoted $\mathrm{C}-\mathrm{H}$ activation of isoureas for the synthesis of 2-alkoxybenzimidazoles

We were pleased to find that the $\mathrm{C}-\mathrm{H}$ activation of isourea $4 a$ can be mediated by $\mathrm{PhI}(\mathrm{OAc})_{2}$ to form $2-(2,2,2-$ trifluoroethoxy)benzimidazole $\mathbf{5 a}$ in $91 \%$ yield at room temperature. The mild reaction conditions of this protocol mean that it could potentially have wide application.

Control experiments were also carried out for mechanistic studies. Nucleophilic addition of $p$-tolylcyanamide (1a) and 2,2,2-trifluoroethanol (3a) did not take place under the optimal conditions, with most of the p-tolylcyanamide being recovered (Scheme $5 a$ ).

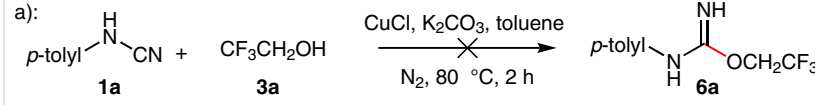

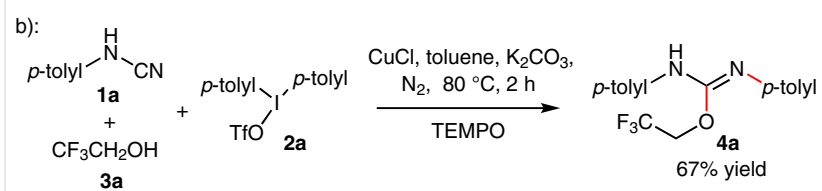

Scheme 5 Control experiments for mechanistic insight

In addition, the nucleophilic addition product $\mathbf{6}$ was a not detected during the three-component reaction. Together, these results suggest that the reaction pathway involving nucleophilic addition of cyanamide $\mathbf{1}$ and fluoroalcohol $\mathbf{3}$ followed by $\mathrm{C}-\mathrm{N}$ coupling with diaryliodonium triflate $\mathbf{2}$ is unlikely (Scheme 6, a).

$$
\text { a) Pathway a: }
$$

b) Pathway b:

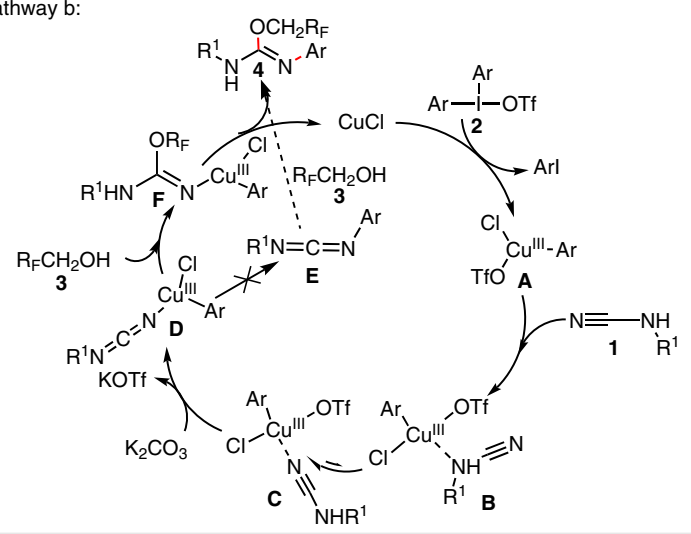

Scheme 6 Proposed reaction mechanism

The addition of the radical inhibitor 2,2,6,6-tetramethylpiperidine-1-oxyl (TEMPO) did not reduce product formation (Scheme $5, \mathrm{~b}$ ), ruling out a radical process. ${ }^{12}$ Thus, a plausible pathway is proposed (Scheme $6, \mathrm{~b}$ ), involving oxidative addition of diaryliodonium triflate $\mathbf{2}$ with $\mathrm{CuCl}^{13}$ followed by coordination with cyanamide $\mathbf{1}$ and isomerisation promoted by $\mathrm{K}_{2} \mathrm{CO}_{3}$ to form intermediate $\mathbf{D}$. Then, $\mathbf{D}$ may undergo reductive elimination and nucleophilic addition with fluoroalcohol $\mathbf{3}$ to generate the desired product $\mathbf{4}$ via intermediate $\mathbf{E}$ or $\mathbf{F}$. The N-arylation products of diarylcyanamides have been proposed as intermediates for the copper-catalysed, three-component reaction of diarylcyanamides, diaryliodoniums and $\mathrm{H}_{2} \mathrm{O} \cdot{ }^{14}$ However, the N-arylation product $\mathbf{E}$ is barely detected in this reaction. Thus, it is reasonable to conclude that the desired product is produced via intermediate $\mathbf{F}$.

In summary, an efficient cooper-catalysed, three-component reaction of cyanamide, fluoroalcohol and diaryliodonium triflate has been developed for the synthesis of $O$-fluoroalkylisoureas in good yields. The use of simple and readily available starting materials is a major practical advantage of this protocol. In addition, the $\mathrm{PhI}(\mathrm{OAc})_{2}$-promoted $\mathrm{C}-\mathrm{H}$ activation of $\mathrm{O}$-fluoroalkoxyisoureas provides a convenient access to potentially valuable 2 -fluoroalkoxybenzimidazoles. Further exploration of such three-component reactions to expand the diversity of this methodology is under way in our laboratory. 
${ }^{1} \mathrm{H}$ NMR spectra were recorded at $400 \mathrm{MHz}$ or $500 \mathrm{MHz}$ with a Bruker AC-500 spectrometer. ${ }^{13} \mathrm{C}$ NMR spectra were similarly recorded at 101 $\mathrm{MHz}$ or $125 \mathrm{MHz}$. Chemical shifts $(\delta)$ are reported in parts per million (ppm) relative to residual proton signals in $\mathrm{CDCl}_{3}(\delta=7.26$, $77.00 \mathrm{ppm}$ ). Coupling constants $(J)$ are reported in Hertz (Hz) and refer to apparent multiplicities; the following abbreviations are used: $s$ (singlet), d (doublet), t (triplet), q (quartet), quin (quintet), sept (septet), hept (heptet), $m$ (multiplet), br (broad signal). Because of the presence of tautomers, we found that some ${ }^{13} \mathrm{C}$ NMR signals were barely detectable for some isoureas, guanidines, ${ }^{14}$ and isothioureas; ${ }^{15}$ only clear signals are reported. Additionally, the total number of ${ }^{13} \mathrm{C}$ NMR signals is more than the total number of non-equivalent carbon atoms in some isoureas, which may be due to the presence of $E / Z$ isomers. Mass spectra were obtained either from an LCMS-IT-TOF (ESI or $\mathrm{APCI}$ ) using positive or negative electron spray $\left(\mathrm{ES}^{+}\right.$or $\mathrm{ES}^{-}$), or from high-resolution mass spectra (HRMS). Flash chromatography was performed using SDS silica gel $60(35-70 \mu \mathrm{m})$. Preparative thin-layer chromatography (TLC) was carried out on $20 \times 20 \mathrm{~cm}$ plates with a layer thickness of 0.5 or $1 \mathrm{~mm}$ (SDS Silicage $160 \mathrm{~F} 254$ ).

All reagents were obtained from commercial suppliers unless otherwise stated. When necessary, organic solvents were routinely dried and/or distilled prior to use and stored over molecular sieves under argon. The starting cyanamides ${ }^{16}$ and diaryliodonium triflates ${ }^{17}$ are known and were prepared according to the reported procedures.

\section{Synthesis of 0-Fluoroalkylisoureas through Copper-Catalysed, Three-Component Reaction of Cyanamides, Fluoroalcohols and Diaryliodonium Triflates; General Procedure}

To a round-bottom sidearm flask, $\mathrm{CuCl}(0.01 \mathrm{mmol}, 0.05$ equiv), cyanamide 1 ( $0.3 \mathrm{mmol}, 1.5$ equiv), diaryliodonium triflate $\mathbf{2}(0.3 \mathrm{mmol}$, 1.5 equiv), and $\mathrm{K}_{2} \mathrm{CO}_{3}(0.45 \mathrm{mmol}, 2.25$ equiv) were sequentially added, and the vessel was degassed and backfilled with nitrogen (balloon). Toluene ( $1 \mathrm{~mL})$ and fluoroalcohol $3(0.2 \mathrm{mmol}, 1$ equiv) were added and the mixture was heated to $80^{\circ} \mathrm{C}$, with stirring for $2 \mathrm{~h}$. The mixture was cooled, the reaction was quenched with water and the mixture was extracted with EtOAc. The organic layers were combined, dried over $\mathrm{MgSO}_{4}$, filtered and concentrated under reduced pressure to give a residue that was purified by preparative $\operatorname{TLC}\left(\mathrm{SiO}_{2}\right)$ to obtain $O$-fluoroalkylisourea 4.

\section{2,2,2-Trifluoroethyl $N, N^{\prime}$-Di-p-tolylcarbamimidate (4a)}

Yield: $42 \mathrm{mg}$ (65\%); colourless waxy solid.

${ }^{1} \mathrm{H} \mathrm{NMR}\left(400 \mathrm{MHz}, \mathrm{CDCl}_{3}\right): \delta=7.18(\mathrm{~d}, J=7.1 \mathrm{~Hz}, 2 \mathrm{H}), 7.08(\mathrm{~d}, J=$ $7.3 \mathrm{~Hz}, 2 \mathrm{H}), 7.98$ (d, $J=7.4 \mathrm{~Hz}, 2 \mathrm{H}), 7.87(\mathrm{~d}, J=7.2 \mathrm{~Hz}, 2 \mathrm{H}), 5.90(\mathrm{br}$, $\mathrm{NH}), 4.76(\mathrm{q}, J=8.6 \mathrm{~Hz}, 2 \mathrm{H}), 2.39-2.27(\mathrm{~m}, 6 \mathrm{H})$.

${ }^{13} \mathrm{C}$ NMR (101 MHz, $\left.\mathrm{CDCl}_{3}\right): \delta=148.66,143.98,134.98,133.49$, $132.88,130.41,129.48,122.16,123.40$ (q, $J=277.2 \mathrm{~Hz}), 121.12,62.52$ (q, $J=36.4 \mathrm{~Hz}), 20.76,20.68$.

IR: 3405.37, 2923.81, 1672.99, 1610.85, 1509.22, 1358.83, 1270.01, $1165.46,1101.68,820.81 \mathrm{~cm}^{-1}$.

HRMS (ESI): $m / z[M-H]^{-}$calcd for $\mathrm{C}_{17} \mathrm{H}_{16} \mathrm{~F}_{3} \mathrm{~N}_{2} \mathrm{O}$ : 321.1220; found: 321.1221.

\section{2,2,2-Trifluoroethyl $N$-Phenyl- $N^{\prime}$-(p-tolyl)carbamimidate (4b)}

Yield: $40 \mathrm{mg}$ (65\%) from phenylcyanamide and di(p-tolyl)iodonium triflate; $41 \mathrm{mg}$ (67\%) from p-tolylcyanamide with diphenyliodonium triflate; colourless waxy solid.

${ }^{1} \mathrm{H}$ NMR $\left(400 \mathrm{MHz}, \mathrm{CDCl}_{3}\right): \delta=6.81-7.36(\mathrm{~m}, 9 \mathrm{H}), 5.93-5.97(\mathrm{~m}, \mathrm{NH})$, $4.79(\mathrm{q}, J=8.5 \mathrm{~Hz}, 2 \mathrm{H}), 2.33(\mathrm{~s}, 3 \mathrm{H})$.
${ }^{13} \mathrm{C}$ NMR $\left(101 \mathrm{MHz}, \mathrm{CDCl}_{3}\right): \delta=148.33,129.84,128.98,124.46$, $123.75,123.38(\mathrm{q}, J=277.7 \mathrm{~Hz}), 122.99,122.34,121.55,120.84$, $119.13,117.88,62.60(q, J=36.3 \mathrm{~Hz}), 21.34$.

IR: 3403.27, 2964.35, 2925.31, 1674.50, 1592.52, 1497.72, 1413.23, 1362.41, 1270.90, 1167.92, 1107.64, 766.34, $697.09 \mathrm{~cm}^{-1}$.

HRMS (ESI): $m / z$ [M-H] $]^{-}$calcd for $\mathrm{C}_{16} \mathrm{H}_{14} \mathrm{~F}_{3} \mathrm{~N}_{2} \mathrm{O}$ : 307.1064; found: 307.1062 .

\section{2,2,2-Trifluoroethyl $N^{\prime}$-Phenyl- $N$-(m-tolyl)carbamimidate (4c)}

Yield: $39 \mathrm{mg}$ (63\%); colourless oil.

$\left.{ }^{1} \mathrm{H} \mathrm{NMR} \mathrm{(400} \mathrm{MHz,} \mathrm{CDCl}_{3}\right): \delta=7.28-7.36(\mathrm{~m}, 2 \mathrm{H}), 6.81-7.17(\mathrm{~m}, 7 \mathrm{H})$, 5.92-5.97 (m, NH), $4.79(\mathrm{q}, J=8.5 \mathrm{~Hz}, 2 \mathrm{H}), 2.33(\mathrm{~s}, 3 \mathrm{H})$.

${ }^{13} \mathrm{C}$ NMR $\left(101 \mathrm{MHz}, \mathrm{CDCl}_{3}\right): \delta=148.32,146.55,139.78,138.89$, 137.54, 137.39, 129.82, 129.64, 128.98, 124.46, 123.73, 123. 58, 123.39 (q, $J=279.6 \mathrm{~Hz}), 123.03,122.35,121.55,120.84,119.18$, $117.88,62.60(\mathrm{q}, J=36.3 \mathrm{~Hz}), 21.34$.

IR: $3407.99,2925.75,1673.74,1592.44,1362.03,1270.35,1107.32$, $696.42 \mathrm{~cm}^{-1}$.

HRMS (ESI): $m / z$ [M-H] $]^{-}$calcd for $\mathrm{C}_{16} \mathrm{H}_{14} \mathrm{~F}_{3} \mathrm{~N}_{2} \mathrm{O}$ : 307.1064; found: 307.1064 .

\section{2,2,2-Trifluoroethyl $N$-Phenyl- $N$-(o-tolyl)carbamimidate (4d)}

Yield: $47 \mathrm{mg}$ (76\%); colourless oil.

${ }^{1} \mathrm{H}$ NMR (400 MHz, $\left.\mathrm{CDCl}_{3}\right): \delta=6.93-7.42(\mathrm{~m}, 9 \mathrm{H}), 5.80-5.86(\mathrm{~m}, \mathrm{NH})$, 4.82-4.90 (m, $2 \mathrm{H}), 2.16-2.26(\mathrm{~m}, 3 \mathrm{H})$.

${ }^{13} \mathrm{C}$ NMR $\left(101 \mathrm{MHz}, \mathrm{CDCl}_{3}\right): \delta=147.76,144.78,137.48,128.97$, $123.83,123.71,123.36$ (q, $J=277.4 \mathrm{~Hz}), 122.31,121.98,120.94$, $119.20,62.43(\mathrm{q}, J=36.4 \mathrm{~Hz}), 17.67$.

IR: 3450.15, 2925.72, 1674.72, 1595.07, 1498.11, 1361.69, 1270.72, $1166.90,1103.05,747.11 \mathrm{~cm}^{-1}$.

HRMS (ESI): $m / z[\mathrm{M}+\mathrm{H}]^{+}$calcd for $\mathrm{C}_{16} \mathrm{H}_{16} \mathrm{~F}_{3} \mathrm{~N}_{2} \mathrm{O}$ : 309.1209; found: 309.1208.

\section{2,2,2-Trifluoroethyl $N$-(2-Fluorophenyl)- $N^{\prime}$-phenylcarbamimida- tez (4e)}

Yield: $39 \mathrm{mg}$ (60\%); pale-yellow oil.

${ }^{1} \mathrm{H}$ NMR (400 MHz, $\mathrm{CDCl}_{3}$ ): $\delta=6.97-7.46(\mathrm{~m}, 8 \mathrm{H}), 6.19(\mathrm{br}, 0.35 \mathrm{H})$, $5.82(\mathrm{br}, 0.59 \mathrm{H}), 4.78(\mathrm{q}, J=8.4 \mathrm{~Hz}, 2 \mathrm{H}), 2.32(\mathrm{~s}, 3 \mathrm{H})$.

${ }^{13} \mathrm{C}$ NMR $\left(101 \mathrm{MHz}, \mathrm{CDCl}_{3}\right): \delta=149.23,134.54,134.18,130.53$, $129.55,124.98,124.45,123.38$ (q, $J=276.7 \mathrm{~Hz}), 122.95,121.92$, $116.68,116.48,115.30,62.86(\mathrm{q}, J=36.5 \mathrm{~Hz}), 20.80$.

IR: $3411.56,2925.80,1674.91,1610.99,1513.44,1415.46,1363.78$, $1272.02,1167.65,1103.94,982.73,753.19 \mathrm{~cm}^{-1}$.

HRMS (ESI): $m / z$ [M $-\mathrm{H}]^{-}$calcd for $\mathrm{C}_{16} \mathrm{H}_{13} \mathrm{~F}_{4} \mathrm{~N}_{2} \mathrm{O}$ : 325.0969; found: 325.0971 .

\section{2,2,2-Trifluoroethyl $N$-(4-Methoxyphenyl)- $N$ '-phenylcarbamim- idate (4f)}

Yield: $41 \mathrm{mg}$ (63\%); pale-yellow waxy solid.

${ }^{1} \mathrm{H} \mathrm{NMR}\left(400 \mathrm{MHz}, \mathrm{CDCl}_{3}\right): \delta=7.26-7.36(\mathrm{~m}, 2 \mathrm{H}), 6.83-6.70(\mathrm{~m}, 7 \mathrm{H})$, 5.83-5.97 (m, NH), 4.46 (q, J = 7.6 Hz, $2 \mathrm{H}), 3.79$ (s, $3 \mathrm{H}$ ).

${ }^{13} \mathrm{C}$ NMR $\left(101 \mathrm{MHz}, \mathrm{CDCl}_{3}\right): \delta=156.57,155.99,148.8,146.79,139.46$, $137.60,130.35,129.79,128.99,123.61,123.44,123.37$ (q, $J=$ $278.0 \mathrm{~Hz}), 123.15,122.42,121.99,120.72,115.16,114.11,62.52(\mathrm{q}, J=$ $36.6 \mathrm{~Hz}), 55.42$. 
IR: $3403.48,2931.00,1670.85,1512.07,1362.57,1269.39,1238.63$, $1164.61,1101.75,833.63 \mathrm{~cm}^{-1}$.

HRMS (ESI): $m / z$ [M - H] $]^{-}$calcd for $\mathrm{C}_{16} \mathrm{H}_{14} \mathrm{~F}_{3} \mathrm{~N}_{2} \mathrm{O}_{2}$ : 323.1013, found: 323.1016.

\section{2,2,2-Trifluoroethyl $N$-Naphthalen-1-yl- $N$ '-(p-tolyl)carbamimidate (4g)}

Yield: $64 \mathrm{mg}$ (89\%) colourless waxy solid.

${ }^{1} \mathrm{H}$ NMR $\left(400 \mathrm{MHz}, \mathrm{CDCl}_{3}\right): \delta=8.09(\mathrm{~d}, J=7.5 \mathrm{~Hz}, 1 \mathrm{H}), 7.90(\mathrm{~d}, J=$ $7.5 \mathrm{~Hz}, 1 \mathrm{H}), 7.66(\mathrm{~d}, J=8.2 \mathrm{~Hz}, 1 \mathrm{H}), 7.47-7.54(\mathrm{~m}, 3 \mathrm{H}), 7.07-7.11(\mathrm{~m}$, $3 \mathrm{H}), 6.95$ (d, $J=7.8 \mathrm{~Hz}, 2 \mathrm{H}), 5.94(\mathrm{br}, \mathrm{NH}), 5.02(\mathrm{q}, J=8.3 \mathrm{~Hz}, 2 \mathrm{H}$ ), $2.32(\mathrm{~s}, 3 \mathrm{H})$.

${ }^{13} \mathrm{C}$ NMR (101 MHz, $\left.\mathrm{CDCl}_{3}\right): \delta=148.56,142.95,134.75,134.68$, $133.74,129.44,128.09,128.04,126.39,126.28,125.69,123.59$, $123.44(\mathrm{q}, J=277.7 \mathrm{~Hz}), 123.39,121.43,117.03,62.56(\mathrm{q}, J=36.1 \mathrm{~Hz})$, 20.65.

IR: 3397.33, 3054.40, 1669.56, 1515.93, 1362.69, 1271.04, 1165.09, $972.13,775.92 \mathrm{~cm}^{-1}$.

HRMS (ESI): $m / z$ [M - H] $]^{-}$calcd for $\mathrm{C}_{20} \mathrm{H}_{17} \mathrm{~F}_{3} \mathrm{~N}_{2} \mathrm{O}$ : 357.1220; found: 357.1217.

\section{2,2,2-Trifluoroethyl $N$-Benzyl- $N$ '-phenylcarbamimidate (4h)}

Yield: $15 \mathrm{mg}$ (24\%); colourless oil.

${ }^{1} \mathrm{H}$ NMR (400 MHz, $\mathrm{CDCl}_{3}$ ): $\delta=7.21-7.35(\mathrm{~m}, 7 \mathrm{H}), 7.03(\mathrm{t}, J=7.3 \mathrm{~Hz}$, $1 \mathrm{H}), 6.88(\mathrm{~d}, J=7.6 \mathrm{~Hz}, 2 \mathrm{H}), 4.68(\mathrm{q}, J=8.5 \mathrm{~Hz}, 2 \mathrm{H}), 4.44(\mathrm{br}, \mathrm{NH})$, $4.33(\mathrm{~s}, 2 \mathrm{H})$.

${ }^{13} \mathrm{C}$ NMR $\left(101 \mathrm{MHz}, \mathrm{CDCl}_{3}\right): \delta=150.94,147.19,138.63,129.68$, $128.64,127.46,127.26,123.43$ (q, $J=276.0 \mathrm{~Hz}$ ), 123.07, 122.48, $122.05,119.33,62.47$ (q, J = 36.2 Hz), 45.68 .

IR: $3423.05,2926.02,1671.45,1593.31,1413.82,1269.32,1164.93$, $1120.28,698.07 \mathrm{~cm}^{-1}$.

HRMS (ESI): $m / z$ [M - H] $]^{-}$calcd for $\mathrm{C}_{16} \mathrm{H}_{14} \mathrm{~F}_{3} \mathrm{~N}_{2} \mathrm{O}$ : 307.1064; found: 307.1067.

\section{2,2,2-Trifluoroethyl $N$-Cyclohexyl- $N$ '-phenylcarbamimidate (4i)}

Yield: $12 \mathrm{mg}$ (20\%); colourless oil.

${ }^{1} \mathrm{H} \mathrm{NMR}\left(400 \mathrm{MHz}, \mathrm{CDCl}_{3}\right): \delta=7.23-7.30(\mathrm{~m}, 2 \mathrm{H}), 7.00(\mathrm{t}, J=7.3 \mathrm{~Hz}$, $1 \mathrm{H}), 6.84(\mathrm{~d}, J=7.1 \mathrm{~Hz}, 2 \mathrm{H}), 4.80-4.52(\mathrm{~m}, 2 \mathrm{H}), 3.88(\mathrm{br}, \mathrm{NH}), 3.42(\mathrm{~s}$, $1 \mathrm{H}), 1.85(\mathrm{~d}, J=11.0 \mathrm{~Hz}, 2 \mathrm{H}), 1.53-1.65(\mathrm{~m}, 3 \mathrm{H}), 1.23-1.29(\mathrm{~m}, 3 \mathrm{H})$, $0.98-1.08(\mathrm{~m}, 2 \mathrm{H})$.

${ }^{13} \mathrm{C}$ NMR $\left(101 \mathrm{MHz}, \mathrm{CDCl}_{3}\right): \delta=151.13,147.59,129.63,123.55(\mathrm{q}, J=$ $276.7 \mathrm{~Hz}), 122.82,122.51,62.27(\mathrm{q}, J=35.9 \mathrm{~Hz}), 50.58,33.77,25.40$, 24.73.

IR: $3420.90,2931.61,2855.92,1669.28,1269.72,1166.65,762.41 \mathrm{~cm}^{-}$ 1 .

HRMS (ESI): $m / z[\mathrm{M}+\mathrm{H}]^{+}$calcd for $\mathrm{C}_{15} \mathrm{H}_{20} \mathrm{~F}_{3} \mathrm{~N}_{2} \mathrm{O}$ : 301.1522; found: 301.1519 .

\section{2,2,2-Trifluoroethyl $N$-(4-Chlorophenyl)- $N^{\prime}$-(p-tolyl)carbam-} imidate (4k)

Yield: $42 \mathrm{mg}$ (62\%); colourless waxy solid.

${ }^{1} \mathrm{H} \mathrm{NMR}\left(400 \mathrm{MHz}, \mathrm{CDCl}_{3}\right): \delta=6.78-7.42(\mathrm{~m}, 8 \mathrm{H}), 5.81-5.93(\mathrm{~m}, \mathrm{NH})$, $4.75(\mathrm{q}, J=8.2 \mathrm{~Hz}, 1.83 \mathrm{H}), 4.56(\mathrm{q}, J=8.0 \mathrm{~Hz}, 0.13 \mathrm{H}), 2.32(\mathrm{~s}, 3 \mathrm{H})$.

${ }^{13} \mathrm{C}$ NMR $\left(101 \mathrm{MHz}, \mathrm{CDCl}_{3}\right): \delta=148.81,145.28,130.48,129.84$, $129.54,129.98,123.77,123.29$ (q, $J=277.4 \mathrm{~Hz}), 122.02,121.38,62.63$ (q, $J=36.5 \mathrm{~Hz}$ ), 20.70 .
IR: $3404.79,2925.49,1672.80,1492.03,1360.44,1270.70,1167.44$, 1096.49, $824.27 \mathrm{~cm}^{-1}$.

HRMS (ESI): $m / z$ [M - H] $]^{-}$calcd for $\mathrm{C}_{14} \mathrm{H}_{13} \mathrm{ClF}_{3} \mathrm{~N}_{2} \mathrm{O}$ : 341.0674; found: 341.0671 .

\section{2,2,2-Trifluoroethyl $N^{\prime}$-(4-Bromophenyl)- $N$-(p-tolyl)carbamim-} idate (4l)

Yield: $27 \mathrm{mg}$ (35\%); colourless waxy solid.

${ }^{1} \mathrm{H} \mathrm{NMR}\left(400 \mathrm{MHz}, \mathrm{CDCl}_{3}\right): \delta=7.39-7.45(\mathrm{~m}, 2 \mathrm{H}), 6.71-7.20(\mathrm{~m}, 6 \mathrm{H})$, $5.80-5.92(\mathrm{~m}, \mathrm{NH}), 4.75(\mathrm{q}, J=8.3 \mathrm{~Hz}, 1.79 \mathrm{H}), 4.55(\mathrm{q}, J=8.2 \mathrm{~Hz}$, $0.11 \mathrm{H}), 2.31(\mathrm{~s}, 3 \mathrm{H})$.

${ }^{13} \mathrm{C}$ NMR $\left(101 \mathrm{MHz}, \mathrm{CDCl}_{3}\right): \delta=148.66,132.79,131.93,130.41$, $129.55,124.23,123.24(\mathrm{q}, J=279.0 \mathrm{~Hz}), 122.32,121.91,121.41$, 116.34, 62.66 (q, $J=36.4 \mathrm{~Hz}), 20.74$.

IR: $3407.63,2924.93,1672.11,1513.56,1488.70,1359.04,1269.88$, $1166.53,821.60 \mathrm{~cm}^{-1}$.

HRMS (ESI): $m / z[\mathrm{M}+\mathrm{H}]^{+}$calcd for $\mathrm{C}_{14} \mathrm{H}_{15} \mathrm{BrF}_{3} \mathrm{~N}_{2} \mathrm{O}$ : 387.0314; found: 387.0317.

2,2,2-Trifluoroethyl $N^{\prime}$-(4-(tert-Butyl)phenyl)- $N$-(p-tolyl)carbamimidate (4m)

Yield: $40 \mathrm{mg}$ (55\%); colourless oil.

${ }^{1} \mathrm{H}$ NMR (400 MHz, $\left.\mathrm{CDCl}_{3}\right): \delta=7.30-7.37(\mathrm{~m}, 2 \mathrm{H}), 7.08-7.16(\mathrm{~m}, 2 \mathrm{H})$, 6.87-6.98 (m, $4 \mathrm{H}), 5.93$ (br, NH), 4.77 (q, $J=8.0 \mathrm{~Hz}, 2 \mathrm{H}$ ), 2.31-2.34 (m, $3 \mathrm{H}), 1.32(\mathrm{~s}, 9 \mathrm{H})$.

${ }^{13} \mathrm{C}$ NMR $\left(101 \mathrm{MHz}, \mathrm{CDCl}_{3}\right): \delta=148.56,130.40,129.48,126.67$, $125.84,122.15,121.84,121.26,120.31,62.51$ (q, $J=35.9 \mathrm{~Hz}), 34.26$, $31.38,20.74$.

IR: $3405.39,2962.96,1673.61,1609.49,1513.14,1361.23,1270.55$, 1166.57, $1103.66,824.92 \mathrm{~cm}^{-1}$.

HRMS (ESI): $m / z$ [M - H] $]^{-}$calcd for $\mathrm{C}_{20} \mathrm{H}_{22} \mathrm{~F}_{3} \mathrm{~N}_{2} \mathrm{O}: 363.1690$; found: 363.1693.

2,2,2-Trifluoroethyl $N^{\prime}$-(2,5-Dimethylphenyl)- $N$-(p-tolyl)carbamimidate (4n)

Yield: $51 \mathrm{mg}$ (76\%); colourless oil.

${ }^{1} \mathrm{H}$ NMR $\left(400 \mathrm{MHz}, \mathrm{CDCl}_{3}\right): \delta=6.73-7.15(\mathrm{~m}, 7 \mathrm{H}), 5.75(\mathrm{br}, \mathrm{NH}), 4.81-$ 4.87 (m, $2 \mathrm{H}), 2.33$ (s, $3 \mathrm{H}), 3.32$ (s, $3 \mathrm{H}), 2.08-2.17$ (m, $3 \mathrm{H})$.

${ }^{13} \mathrm{C}$ NMR $\left(101 \mathrm{MHz}, \mathrm{CDCl}_{3}\right): \delta=147.70,144.76,136.92,135.0,133.64$, $130.95,130.43,129.54,127.34,124.41,122.47,122.14,121.38,62.39$ (q, $J=36.4 \mathrm{~Hz}$ ), 21.02, 20.74, 17.27.

IR: $3408.34,2924.07,1673.26,1611.24,1514.20,1358.85,1270.39$, $1166.19,811.07 \mathrm{~cm}^{-1}$.

HRMS (ESI): $m / z$ [M - H] $]^{-}$calcd for $\mathrm{C}_{18} \mathrm{H}_{18} \mathrm{~F}_{3} \mathrm{~N}_{2} \mathrm{O}$ : 335.1377; found: 335.1378 .

\section{2,2,2-Trifluoroethyl $N$ '-Mesityl- $N$-(p-tolyl)carbamimidate (40)}

Yield: $51 \mathrm{mg}$ (73\%); colourless oil.

${ }^{1} \mathrm{H}$ NMR (400 MHz, $\left.\mathrm{CDCl}_{3}\right): \delta=7.07-7.17(\mathrm{~m}, 2 \mathrm{H}), 6.85-6.96(\mathrm{~m}, 4 \mathrm{H})$, $5.57(\mathrm{br}, 0.74 \mathrm{H}), 5.32(\mathrm{br}, 0.23 \mathrm{H}), 4.89(\mathrm{q}, J=8.4 \mathrm{~Hz}, 1.52 \mathrm{H}), 4.70(\mathrm{q}$, $J=8.1 \mathrm{~Hz}, 0.44 \mathrm{H}), 2.29-2.34(\mathrm{~m}, 6 \mathrm{H}), 2.14-2.18(\mathrm{~m}, 6 \mathrm{H})$.

${ }^{13} \mathrm{C}$ NMR $\left(101 \mathrm{MHz}, \mathrm{CDCl}_{3}\right): \delta=147.01,140.48,134.91,133.58$, $132.53,129.54,129.20,127.56,124.80,123.42(\mathrm{q}, J=278.6 \mathrm{~Hz})$, 122.26, 121.44, 119.28, 62.20 (q, $J=36.4 \mathrm{~Hz}$ ), 20.68, 20.66, 17.97, 17.80 . 
IR: 3396.09, 2922.11, 1675.51, 1611.58, 1514.45, 1357.22, 1271.30, 1167.24, 1104.41, 981.66, $820.59 \mathrm{~cm}^{-1}$.

HRMS (ESI): $m / z$ [M - H] $]^{-}$calcd for $\mathrm{C}_{19} \mathrm{H}_{20} \mathrm{~F}_{3} \mathrm{~N}_{2} \mathrm{O}$ : 349.1533; found: 349.1535 .

2,2,2-Trifluoroethyl $N^{\prime}$-(4-(tert-Butyl)phenyl)- $N$-(p-tolyl)carbamimidate / 2,2,2-Trifluoroethyl $N$-Phenyl- $N$ '-(p-tolyl)carbamimidate $\left(4 \mathrm{~m} / 4 \mathrm{~m}^{\prime}\right)$

Yield: $44 \mathrm{mg}$ (64\%); $\mathbf{4 m} / \mathbf{4 m}$ '=1.5:1; pale-yellow oil.

${ }^{1} \mathrm{H}$ NMR $\left(400 \mathrm{MHz}, \mathrm{CDCl}_{3}\right): \delta=7.30-7.34(\mathrm{~m}, 2.95 \mathrm{H}), 7.89-7.17(\mathrm{~m}$, $10.23 \mathrm{H}$ ), 5.94 (br, $1.54 \mathrm{H}), 4.78$ (m, $3.12 \mathrm{H}), 2.33$ (br, $5.42 \mathrm{H}), 1.33$ (s, $9 \mathrm{H})$.

${ }^{13} \mathrm{C}$ NMR (101 MHz, $\left.\mathrm{CDCl}_{3}\right): \delta=148.54,143.83,134.99,129.83$, $128.98,125.83,124.76,122.40,122.05,121.24,120.29,62.57$ (q, $J=$ $36.4 \mathrm{~Hz}), 62.51$ (q, $J=36.4 \mathrm{~Hz}), 34.25,31.36,20.73$.

IR: $3404.90,2963.49,2928.48,1674.19,1607.41,1513.58,1414.69$, 1362.44, 1270.97, 1167.46, 1104.34, 981.13, $825.09 \mathrm{~cm}^{-1}$.

HRMS (ESI): $m / z$ [M - H] $]^{-}$calcd for $\mathrm{C}_{16} \mathrm{H}_{14} \mathrm{~F}_{3} \mathrm{~N}_{2} \mathrm{O}$ : 307.1064; found: 307.1067; m/z [M - H] $]^{-}$calcd for $\mathrm{C}_{20} \mathrm{H}_{22} \mathrm{~F}_{3} \mathrm{~N}_{2} \mathrm{O}$ : 363.1690; found: 363.1693 .

2,2,2-Trifluoroethyl $N^{\prime}$-(4-Iodophenyl)- $N$-( $p$-tolyl)carbamimidate / 2,2,2-Trifluoroethyl $N$-Phenyl- $N^{\prime}$-(p-tolyl)carbamimidate (4p/4p')

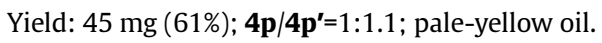

${ }^{1} \mathrm{H}$ NMR $\left(400 \mathrm{MHz}, \mathrm{CDCl}_{3}\right): \delta=7.57-7.64(\mathrm{~m}, 0.88 \mathrm{H}), 7.28-7.36(\mathrm{~m}$, $0.95 \mathrm{H}), 7.67-7.15$ (m, $6.39 \mathrm{H}), 5.80-5.94(\mathrm{~m}, 1 \mathrm{H}), 4.72-4.80$ (m, $2 \mathrm{H})$, $2.32(\mathrm{~s}, 3 \mathrm{H})$.

${ }^{13} \mathrm{C}$ NMR $\left(101 \mathrm{MHz}, \mathrm{CDCl}_{3}\right): \delta=148.59,146.53,138.82,137.97$, $130.47,129.86,129.59,129.03,124.82,123.58,122.06,121.92$, $121.46,121.29,120.82,86.90,62.68(\mathrm{q}, J=36.0 \mathrm{~Hz}), 62.64(\mathrm{q}, J=$ $36.3 \mathrm{~Hz}), 20.79$.

IR: 3404.56, 3026.19, 2925.05, 2856.81, 1673.06, 1593.32, 1514.54, $1492.04,1414.79,1361.07,1270.28,1166.97,1102.15,980.08$, $812.92 \mathrm{~cm}^{-1}$.

HRMS (ESI): $m / z$ [M $+\mathrm{H}]^{+}$calcd for $\mathrm{C}_{16} \mathrm{H}_{16} \mathrm{~F}_{3} \mathrm{~N}_{2} \mathrm{O}$ : 309.1209; found: 309.1207; $\mathrm{m} / z$ [M $-\mathrm{H}]^{-}$calcd for $\mathrm{C}_{16} \mathrm{H}_{14} \mathrm{~F}_{3} \mathrm{IN}_{2} \mathrm{O}$ : 433.0030; found: 433.0031 .

\section{2-Fluoroethyl $N, N^{\prime}$-Di-p-tolylcarbamimidate (4r)}

Yield: $28 \mathrm{mg}$ (49\%); colourless oil.

${ }^{1} \mathrm{H}$ NMR (400 MHz, $\left.\mathrm{CDCl}_{3}\right): \delta=7.64(\mathrm{~d}, J=8.2 \mathrm{~Hz}, 2 \mathrm{H}), 7.19(\mathrm{~d}, J=$ $8.1 \mathrm{~Hz}, 2 \mathrm{H}), 7.09(\mathrm{~d}, J=7.9 \mathrm{~Hz}, 2 \mathrm{H}), 7.00(\mathrm{~d}, J=7.9 \mathrm{~Hz}, 2 \mathrm{H}), 4.41(\mathrm{t}, J=$ $7.5 \mathrm{~Hz}, 2 \mathrm{H}), 3.99$ (t, J = 7.5 Hz, $2 \mathrm{H}), 2.35$ (s, $3 \mathrm{H}), 2.32$ (s, $3 \mathrm{H})$.

${ }^{13} \mathrm{C}$ NMR $\left(101 \mathrm{MHz}, \mathrm{CDCl}_{3}\right): \delta=149.26,144.84,137.22,132.45$, $131.55,129.28,129.09,122.94,118.92,63.60,46.5,20.79,20.68$.

IR: $3425.30,2920.65,1673.61,1606.30,1508.24,1400.43,1312.80$, 1201.39, 1101.60, 1041.78, $809.95 \mathrm{~cm}^{-1}$.

HRMS (ESI): no molecular ion peak was found.

\section{2,2-Difluoroethyl $\boldsymbol{N}, \boldsymbol{N}^{\prime}$-Di-p-tolylcarbamimidate (4s)}

Yield: $52 \mathrm{mg}$ with di(p-tolylcyanamide) (63\%); pale-yellow waxy solid.

${ }^{1} \mathrm{H} \mathrm{NMR}\left(400 \mathrm{MHz}, \mathrm{CDCl}_{3}\right): \delta=7.11-7.28(\mathrm{~m}, 8 \mathrm{H}), 6.22(\mathrm{tt}, J=55.5$, $3.9 \mathrm{~Hz}, 1 \mathrm{H}), 5.95(\mathrm{br}, \mathrm{NH}), 4.60(\mathrm{td}, J=13.6,3.9 \mathrm{~Hz}, 2 \mathrm{H}), 2.40(\mathrm{~s}, 3 \mathrm{H})$, $2.37(\mathrm{~s}, 3 \mathrm{H})$.
${ }^{13} \mathrm{C}$ NMR $\left(101 \mathrm{MHz}, \mathrm{CDCl}_{3}\right): \delta=149.24,144.25,135.18,133.30$, $132.66,129.41,122.22,115.42,113.03(t, J=240.9 \mathrm{~Hz}), 110.64,64.55$ $(\mathrm{t}, J=29.9 \mathrm{~Hz}), 20.72,20.67$.

IR: 3707.84, 2922.41, 1662.54, 1506.18, 1084.39, $809.14 \mathrm{~cm}^{-1}$. HRMS (ESI): $m / z$ [M $-\mathrm{H}]^{-}$calcd for $\mathrm{C}_{17} \mathrm{H}_{17} \mathrm{~F}_{2} \mathrm{~N}_{2} \mathrm{O}$ : 303.1314; found: 303.1315.

\section{1,1,1,3,3,3-Hexafluoropropan-2-yl $N, N^{\prime}$-Di-p-tolylcarbamimidate (4t)}

Yield: $40 \mathrm{mg}$ (51\%); pale-yellow waxy solid.

${ }^{1} \mathrm{H}$ NMR $\left(400 \mathrm{MHz}, \mathrm{CDCl}_{3}\right): \delta=7.18(\mathrm{~d}, J=7.6 \mathrm{~Hz}, 2 \mathrm{H}), 7.09(\mathrm{~d}, J=$ $7.6 \mathrm{~Hz}, 2 \mathrm{H}), 6.94(\mathrm{~d}, J=31.8,7.9 \mathrm{~Hz}, 2 \mathrm{H}), 6.86(\mathrm{~d}, J=31.8,7.9 \mathrm{~Hz}, 2 \mathrm{H})$, 6.56 (hept, $J=12.8,6.3 \mathrm{~Hz}, 1 \mathrm{H}), 5.94(\mathrm{br}, \mathrm{NH}), 2.35$ (s, $3 \mathrm{H}), 2.31$ (s, $3 \mathrm{H})$.

${ }^{13} \mathrm{C}$ NMR $\left(101 \mathrm{MHz}, \mathrm{CDCl}_{3}\right): \delta=147.30,142.93,134.32,134.17$, 133.36, 130.45, 129.58, 121.94, 121.90, 120.92 (q, J = 280.1 Hz), 67.85 (hept, $J=34.2 \mathrm{~Hz}$ ), 20.79, 20.72 .

IR: $3402.97,2926.74,1684.92,1611.36,1512.87,1357.28,1276.98$, $1230.50,1195.80,1108.88,821.78 \mathrm{~cm}^{-1}$.

HRMS (ESI): $m / z[\mathrm{M}+\mathrm{H}]^{+}$calcd for $\mathrm{C}_{18} \mathrm{H}_{17} \mathrm{~F}_{6} \mathrm{~N}_{2} \mathrm{O}$ : 391.1240; found: 391.1239.

\section{Ethyl $\mathbf{N}, \boldsymbol{N}^{\prime}$-Di-p-tolylcarbamimidate (4u)}

Yield: $18 \mathrm{mg}$ (34\%); colourless waxy solid.

${ }^{1} \mathrm{H}$ NMR $\left(400 \mathrm{MHz}, \mathrm{CDCl}_{3}\right): \delta=7.19-7.01(\mathrm{~m}, 4 \mathrm{H}), 6.88-6.94(\mathrm{~m}, 4 \mathrm{H})$, $5.80(\mathrm{br}, \mathrm{NH}), 4.40(\mathrm{q}, J=7.0 \mathrm{~Hz}, 2 \mathrm{H}), 2.31(\mathrm{~s}, 6 \mathrm{H}), 1.40(\mathrm{t}, J=7.1 \mathrm{~Hz}$, $3 \mathrm{H})$.

${ }^{13} \mathrm{CNMR}\left(101 \mathrm{MHz}, \mathrm{CDCl}_{3}\right): \delta=150.36,136.07,132.53,132.12,130.25$, $129.85,129.32,122.52,120.61,62.57,20.74,20.66,14.32$.

IR: $3412.51,1657.62,1610.38,1510.33,1381.07,1327.68,1232.22$, $1064.68,813.77 \mathrm{~cm}^{-1}$.

HRMS (ESI): $m / z[\mathrm{M}-\mathrm{H}]^{-}$calcd for $\mathrm{C}_{17} \mathrm{H}_{19} \mathrm{~N}_{2} \mathrm{O}$ : 267.1503; found: 267.1504.

\section{Synthesis of 2-Fluoroalkoxybenzimidazoles through $\mathrm{PhI}(\mathrm{OAc})_{2}$ - Promoted C-H Activation of $O$-Fluoroalkyl Isoureas; General Pro- cedure}

To a round-bottom flask, $O$-fluoroalkylisourea $4(0.2 \mathrm{mmol}, 1.0$ equiv), $\mathrm{PhI}(\mathrm{OAc})_{2}(0.4 \mathrm{mmol}, 2$ equiv) and acetonitrile $(3 \mathrm{~mL})$ were added and the mixture was stirred at r.t. for $45 \mathrm{~min}$, then concentrated under reduced pressure to give a residue, which was purified to obtain 2-fluoroalkoxybenzimidazole 5 after preparative $\operatorname{TLC}\left(\mathrm{SiO}_{2}\right)$.

\section{6-Methyl-1-(p-tolyl)-2-(2,2,2-trifluoroethoxy)-1H-benzo[d]imid-} azole (5a)

Yield: $58 \mathrm{mg}$ (91\%); pale-yellow waxy solid.

${ }^{1} \mathrm{H} \mathrm{NMR}\left(400 \mathrm{MHz}, \mathrm{CDCl}_{3}\right): \delta=7.49(\mathrm{~d}, J=8.1 \mathrm{~Hz}, 1 \mathrm{H}), 7.41-7.30(\mathrm{~m}$, $4 \mathrm{H}), 7.07$ (d, J = 8.1 Hz, $1 \mathrm{H}), 7.02(\mathrm{~s}, 1 \mathrm{H}), 4.96$ (q, J = 8.2 Hz, $2 \mathrm{H}), 2.46$ (s, $3 \mathrm{H}), 2.42(\mathrm{~s}, 3 \mathrm{H})$.

${ }^{13} \mathrm{C}$ NMR $\left(101 \mathrm{MHz}, \mathrm{CDCl}_{3}\right): \delta=154.56,138.17,137.05,134.68$, 132.04, 131.45, 130.18, $122.75(\mathrm{q}, J=277.8 \mathrm{~Hz}), 125.65,123.72$, $117.70,109.73,65.77$ (q, $J=37.0 \mathrm{~Hz}), 21.63,21.14$.

IR: 3446.07, 2962.47, 2924.88, 1630.20, 1547.98, 1517.91, 1442.49, 1273.13, 1169.82, 1091.37, 962.70, $808.55 \mathrm{~cm}^{-1}$.

HRMS (ESI): no molecular ion peak was found. 


\section{Funding Information}

The authors acknowledge the Natural Science Foundation of Hainan Province (grant numbers 20162015, 20152027), the National Natural Science Foundation of China (51263006) and Hainan Province International Science and Technology Specific (KJHZ2014-02) for financial support of this work

\section{Acknowledgment}

The Analytical and Testing Center of Hainan University is acknowledged for excellent technical and analytical support.

\section{Supporting Information}

Supporting information for this article is available online at https://doi.org/10.1055/s-0036-1590828.

\section{References}

(1) For selected examples of chain isoureas employed for guanylating and alkylating reagents, see: (a) Knut, K.; Michael, K.; Thomas, G.; Thomas, B.; Günter, S.; Matthlas, R.; Richard van G, EP 1013640B1, 1999. (b) Duffy, M. G.; Grayson, D. H. J. Chem. Soc., Perkin Trans. 1 2002, 1555. (c) Crosignani, S.; White, P. D.; Linclau, B. J. Org. Chem. 2004, 69, 5897. (d) Crosignani, S.; White, P. D.; Linclau, B. Org. Lett. 2002, 4, 1035. (e) Crosignani, S.; White, P. D.; Linclau, B. Org. Lett. 2002, 4, 2961. (f) Crosignani, S.; White, P. D.; Linclau, B. Org. Lett. 2003, 5, 853. (g) Liu, Y. Synlett 2009, 1353.

(2) (a) Shibouta, Y.; Inada, Y.; Ojima, M.; Wada, T.; Noda, M.; Sanada, T.; Kubo, K.; Kohara, Y.; Naka, T.; Nishikawa, K. J. Pharmacol. Exp. Ther. 1993, 266, 114. (b) Garcia-Donaire, J. A.; Ruilope, L. M. Diabetes Res. Clin. Pract. 2007, 76S, S22. (c) Carvalho, L. C. R.; Fernandes, E.; Marques, M. M. B. Chem. Eur. J. 2011, 17, 12544.
(3) Trapero, A.; Alfonso, I.; Butters, T. D.; Llebaria, A. J. Am. Chem. Soc. 2011, 133, 5474.

(4) (a) Lengfeld, F.; Stieglitz, J. Ber. Dtsch. Chem. Ges. 1894, 27, 926. (b) Stieglitz, J. Ber. Dtsch. Chem. Ges. 1895, 28, 573.

(5) Dains, F. B. J. Am. Chem. Soc. 1899, 21, 136.

(6) Däbritz, E. Angew. Chem. Int. Ed. Engl. 1966, 5, 470.

(7) Schmidt, E.; Reichle, A.; Wandel, M.; Carl, W.-R. German Published Pat. Appl 1181262, 1962.

(8) Batrice, R. J.; Kefalidis, C. E.; Maron, L.; Eisen, M. S. J. Am. Chem. Soc. 2016, 138, 2114

(9) (a) Li, J.; Wang, D.; Zhang, Y.; Li, J.; Chen, B. Org. Lett. 2009, 11, 3024. (b) Li, J.; Neuville, L. Org. Lett. 2013, 15, 1752. (c) Tran, L. Q.; Li, J.; Neuville, L. J. Org. Chem. 2015, 80, 6102. (d) Li, J.; Wang, H.; Hou, Y.; Yu, W.; Xu, S.; Zhang, Y. Eur. J. Org. Chem. 2016, 2388.

(10) (a) Organofluorine Chemistry: Principles and Commercial Applications; Banks, R. E.; Smart, B. E.; Tatlow, J. C., Eds.; Plenum Press: New York, 1994. (b) Biomedical Frontiers of Fluorine Chemistry; Ojima, I.; McCarthy, J.; Welch, J., Eds.; American Chemical Society: Washington D. C., 1996. (c) Fluorine-Containing Synthons; Soloshonok, V. A., Ed.; American Chemical Society: Washington D. C., 2005.

(11) Li, J.; Zheng, X.; Li, W.; Zhou, W.; Zhu, W.; Zhang, Y. New J. Chem. 2016, 40, 77.

(12) (a) Xu, J.; Zhang, P.; Gao, Y.; Chen, Y.; Tang, G.; Zhao, Y. J. Org. Chem. 2013, 78, 8176. (b) Carroll, M. A.; Nairne, J.; Smith, G.; Widdowson, D. A. J. Fluorine Chem. 2007, 128, 127.

(13) (a) Phipps, R. J.; Grimster, N. P.; Gaunt, M. J. J. Am. Chem. Soc. 2008, 130, 8172. (b) Phipps, R. J.; Gaunt, M. J. Science 2009, 322, 1593. (c) Cahard, E.; Bremeyer, N.; Gaunt, M. J. Angew. Chem. Int. Ed. 2013, 52, 9284.

(14) Li, P.; Cheng, G.; Zhang, H.; Xu, X.; Gao, J.; Cui, X. J. Org. Chem. 2014, 79, 8156

(15) Mampuys, P.; Zhu, Y.; Vlaar, T.; Ruijter, E.; Orru, R. V. A.; Maes, B. U. W. Angew. Chem. Int. Ed. 2014, 53, 12849.

(16) (a) Sahoo, S. K.; Jamir, L.; Guin, S.; Patel, B. K. Adv. Synth. Catal. 2010, 352, 2538. (b) Kumar, V.; Kaushik, M. P.; Mazumdar, A. Eur. J. Org. Chem. 2008, 1910.

(17) Bielawski, M.; Zhu, M.; Olofsson, B. Adv. Synth. Catal. 2007, 349, 2610. 